\title{
Atomistic Understanding of Interface Structures and Properties in Self-Assembled and Vertically Aligned Nanocomposite Thin Films by Advanced Scanning Transmission Electron Microscopy
}

Ping Lu ${ }^{1}$, Lin Zhou ${ }^{2}$, Jon Ihlefeld ${ }^{3}$ and Wei Pan ${ }^{1}$

${ }^{1}$ Sandia National Laboratories, Albuquerque, NM, USA

2. Ames Laboratory, Ames, IA, USA

3. Department of Materials Science and Engineering, University of Virginia, Charlottesville, VA, USA

The structural and chemical characterization up to atomic-scale plays a critical role in understanding structure-property relationships. Self-assembled, vertically aligned nanocomposite (VAN) thin films, consisting of two immiscible components hetero-epitaxially grown on single crystals, offer the advantage of utilizing the functionalities of both components. The structure also provides the possibility of tuning the material's properties by tailoring the volume ratio of two components, the interface-to-volume ratio, hetero-epitaxial strain and the interface properties [1]. Recent technical advances in scanning transmission electron microscopy (STEM) have made it possible to perform atomic-resolution characterization of the VANs and establish the structure-property relationships [2-4]. Here we describe several efforts of utilizing advanced STEM techniques to quantify the interfaces of VAN thin films including $\mathrm{ZnO}: \mathrm{La}_{0.7} \mathrm{Sr}_{0.3} \mathrm{MnO}_{3}$ (LSMO), and Sm-doped $\mathrm{CeO}_{2}$ (SDC): $\mathrm{SrTiO}_{3}$ (STO). STEM imaging combining low-angle, and highangle annular dark-field (LAADF, HAADF) detectors as well as atomic-scale elemental mapping using energy-dispersive X-ray spectroscopy (EDS) and electron energy loss-spectroscopy (EELS) are used to study cation inter-diffusion across the interfaces, dopant distribution within each component, and $\mathrm{O}$ vacancy and valence state of cations across/near the interfaces.

Fig. 1a shows a typical plan-view STEM HAADF image of ZnO:LSMO VAN grown on $\mathrm{SrTiO}_{3}$ (STO) substrate. The self-assembled $\mathrm{ZnO}$ nanocolumns with an average diameter of $\sim 5 \mathrm{~nm}$ are distributed in the LSMO matrix. A white-contrast, ring-like shell is present around the $\mathrm{ZnO}$ nanopillars in Fig. 1a, signifying the presence of interdiffusion layer between the $\mathrm{ZnO}$ and LSMO. Fig. 1b shows a composite color EDS map, made of $\mathrm{Zn} K$ (red), Sr $K$ (blue), La $L$ (green), and Mn $K$ (cyan) maps. The individual map for each element is also shown in Fig. 1c. These results indicate presence of a (La,Mn)-rich and Sr-deficient shell around the $\mathrm{ZnO}$ pillars. The shell structure is likely responsible for electrical current induced magnetic hysteresis in the composite film. Fig.2a shows the plan-view STEM HAADF image of the SDC:STO VAN. By combining LAADF and HAADF imaging (Figs.2b and 2c), the presence of oxygen vacancy and/or other point defects near the interfaces can be revealed. The results collaborate with enhanced oxygen ion conductivity observed in the structure. The atomic STEM-EDS and STEM-EELS are further applied to study the interfaces [5].

\section{References:}

[1] Yu, P., Chu, Y.-H. \& Ramesh, R., Mater. Today 15 (2012), p. 320.

[2] Von Harrach, H.S. et al, Microsc. Microanal. 15 (suppl.2) (2009), p. 208.

[3] P. Lu et al, Microsc. Microanal. 20 (2014), p. 1782.

[4] P. Lu et al, Sci. Rep. 4 (2014), p. 3945.

[5] Sandia National Laboratories is a multi-mission laboratory managed and operated by National 
Technology and Engineering Solutions of Sandia LLC, a wholly owned subsidiary of Honeywell International Inc., for the U.S. Department of Energy's National Nuclear Security Administration under contract DE-NA0003525. Authors thank Professors Q.X. Jia (University of Buffalo), H.Y. Wang (Purdue University) and J. L. MacManus-Driscoll (University of Cambridge (UK)) for providing the samples necessary for the work.
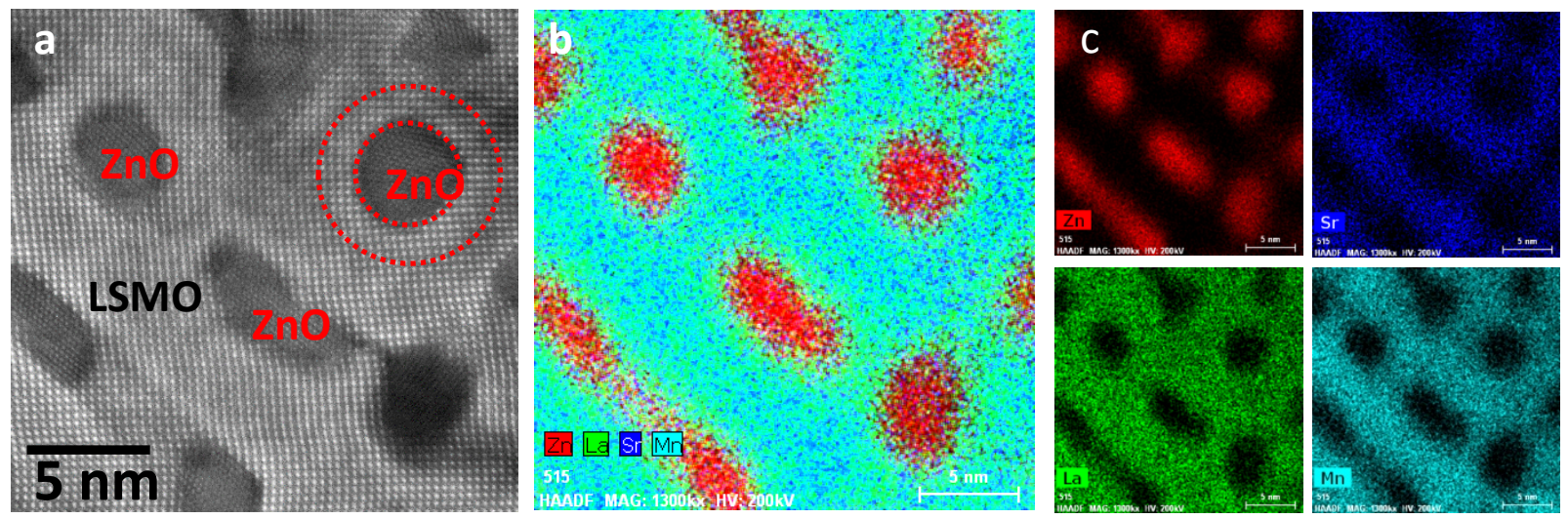

Figure 1. (a) STEM HAADF image and (b, c) EDS maps of ZnO:LSMO VAN, showing the core-shell structure with a (La, Mn)-rich and Sr-deficient composition around the $\mathrm{ZnO}$ pillars.
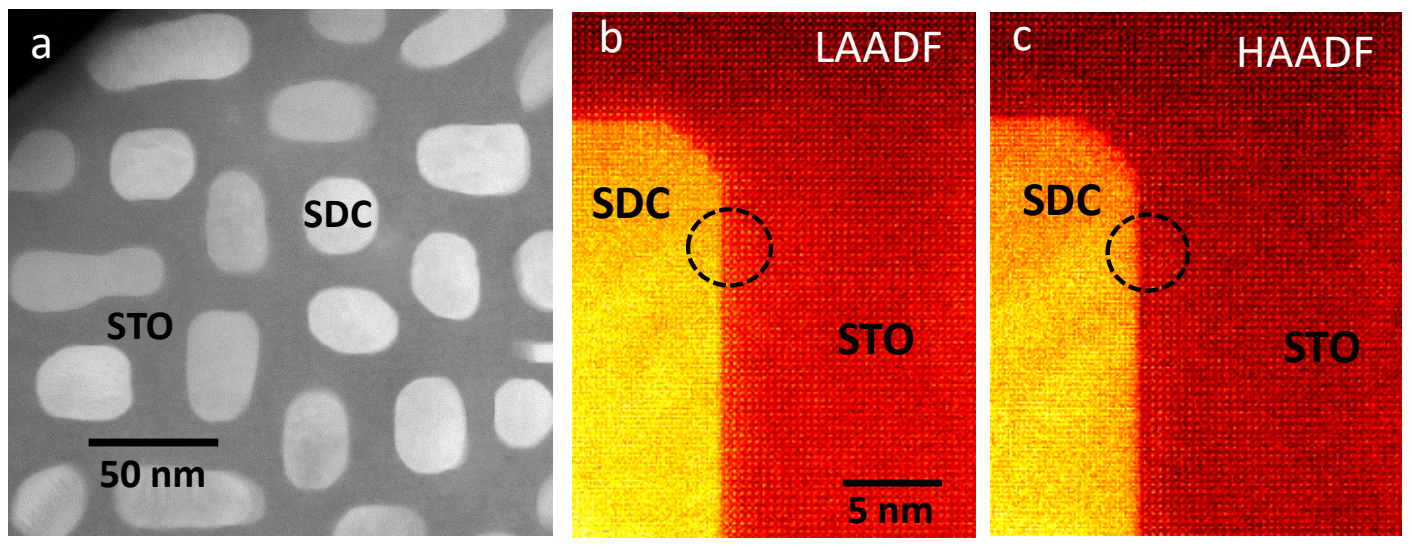

Figure 2. (a) STEM HAADF image of SDC:STO VAN; and (b, c) STEM LAADF and HAADF image, respectively, showing the subtle contrast difference between the images near the interfaces due to presence of oxygen vacancies and/or point defects. 